\title{
Energy Efficient Cluster based Routing Protocol for WSN based on Hybrid BSO-TLBO Optimization Model
}

J Sathya Priya ( $\sim$ jsathya2priya@gmail.com )

Velammal Engineering College https://orcid.org/0000-0001-9237-6328

Wael Mohammad Alenazy

King Saud University

A R Sathyabama

Velammal Engineering College

\section{Research Article}

Keywords: sensor nodes, BSO, TLO, Cluster Head, energy-efficient

Posted Date: June 1st, 2021

DOI: https://doi.org/10.21203/rs.3.rs-188784/v1

License: (c) (i) This work is licensed under a Creative Commons Attribution 4.0 International License.

Read Full License 


\title{
Energy Efficient Cluster based Routing Protocol for WSN based on Hybrid BSO-TLBO Optimization Model
}

\author{
J Sathya Priya $^{1 *}$, Dr. Wael Mohammad Alenazy ${ }^{2}$, A R Sathyabama ${ }^{3}$ \\ ${ }^{* 1}$ Associate Professor, Department of Computer Science and Engineering, \\ Velammal Engineering College, Chennai, India \\ ${ }^{2}$ Assistant Professor, Computer Science, Department of Self Development Skills, \\ CFY Deanship King Saud University, Riyadh, Saudi Arabia \\ ${ }^{3}$ Assistant Professor, Department of Information Technology, \\ Velammal Engineering College, Chennai, India \\ *Corresponding Author Email. Id: jsathya2priya@gmail.com
}

\begin{abstract}
:
The most famous Wireless Sensor Networks (WSN) is one of the cheapest and rapidly evolving networks in modern communication. It can be used to sense various substantial and environmental specifications by providing cost-effective sensor devices. The development of these sensor networks is exploited to provide an energy-efficient weighted clustering method to increase the lifespan of the network. We propose a novel energy-efficient method which utilizes the Brainstorm algorithm in order to adopt the ideal $\mathrm{CH}$ to reduce energy-draining. Further, the effectiveness of the Brain Storm Optimization (BSO) algorithm is enhanced with the incorporation of the Modified Teacher-Learner Optimized (MTLBO) algorithm with it. The modified BSOMTLBO algorithm can be used to attain an improved throughput, network lifetime, and to reduce the energy consumption by nodes and $\mathrm{CH}$, death of sensor nodes, routing overhead. The performance of our proposed work is analyzed with other existing approaches and inference that our approach performs better than all the other approaches.
\end{abstract}

Keywords: sensor nodes, BSO, TLO, Cluster Head, energy-efficient

\section{Introduction:}

Recently, the most common and popular evolving network is Wireless Sensor Network (WSN), which plays a vital role in communication fields because of cheaper sensor devices, low developmental complications, inexpensive with the capability to sense various kinds of physical and environmental considerations also transmitting, processing and sensing information [1]. The physical and environmental conditions such as temperature, acoustic, vibration, and pressure are observed by the spatially distributed independent sensors present in the WSN, which may approve the information via the leading position called base station (BS). WSN comprises many applications in different fields such as industrial, commercial, medical, defense domain, weather forecasting, etc. For various applications, the obtained data are examined to create a decision at BS [2]. The limited energy, power resources with a superior number of nodes is present in the WSNs. More energy is required to pass on sensed data packets to the base station, hence, an energy-efficient routing protocol is an important one.

The data transmission to BS via nodes and environmental sensing parameters are the major ways to exhaust its energy in WSN. The data processing and sensing required lower energy than data transmission. These routing protocols may construct the communication path among both base stations as well as sensor nodes (SN). Nevertheless, one of the crucial work of these sensor nodes is effectively and securely transmit the data from nodes to base stations. Maximum energy, node failure, poor network lifetime, reliability, hardware limitations, fault tolerance, scalability, and so on to affect WSN architecture
[3]. In the past decades, several routing methods were introduced to overcome these shortcomings. The network scalability enhancement, minimization of network energy consumption are achieved via sensors grouping into clusters. The optimal Quality of Service (QoS) parameters are obtained by using a cross-layer technique. The information exchanging in non-hierarchical behavior via different communication protocol layers [4]. According to the network structure, the flat and hierarchical are the different classifications of routing protocols.

Every SN plays a major role to reduce the network overhead, stability, scalability, and energy effectiveness in flat routing protocols [5]. The coherence protocols and querybased protocols are the major classifications of communication model routing protocols. The transmission overhead is minimized with the help of the meta-negotiation technique [6]. The location-based and mobile agent-based algorithms are topology-based protocols. Route failures are effectively handled in the presence of reliable and multipath based routing protocols. The WSN performances in terms of network lifetime, energy, and cost are enhanced through different kinds of methodologies. Besides, different kinds of clustering methods such as fuzzy, k-means, LEACH,Aware Cluster-Based Routing (ACBR) protocol, Gravitation Search Algorithm (GSA), Harmony search algorithm (HSA),Optics inspired optimization (OIO), Simulated Annealing (SA), etc to enhance the SN deficiency [4-7]. These methodologies have few shortcomings in terms of node energy level preservation, cost, packet delay, limited energy in sensor nodes, poor network performance, path selection, reliability, node selection, etc. Hence, we proposed a novel method known as the Energy-efficient weighted clustering method based on the 
BSO-TLBO algorithm for WSN. The major contribution of this work is summarized below:

$>$ The BSO algorithm is exploited to form the clusters of effective sensor nodes. Further to enhance the selection of $\mathrm{CH}$ we incorporate $\mathrm{BSO}$ with the TLBO algorithm. The TLBO algorithm is used to fine-tune the parameters of BSO for a better selection of $\mathrm{CH}$.

$>$ The fitness functions used in the ranking phase are used to improve the energy efficiency of the system. optimal routing is selected by the modified BSO algorithm.

$>$ Throughput, network lifetime, total packets received are increased due to the optimal routing provided by our proposed method. Meanwhile, the energy consumption during transmission of data and by $\mathrm{CH}$ is reduced to a great extent.

The rest of the paper is organized as follows, Section 2 reviews the related works. In section 3 provide information about our proposed methodology, i.e., BSO-TLBO algorithms and their processes. The experimental analysis and its comparative studies are explained in section 4 . To the end with we conclude in section 5 .

\section{Related works:}

The Cluster-Tree-based Energy-Efficient Data Gathering (CTEEDG) method was proposed by Karunanithy et al. [8] to enhance the network throughput as well as a lifetime. Based on the collected information, the Fuzzy logic is to choose the $\mathrm{CH}$ by means of CTEEDG. The congestion-free shortest path to the BS is ensured thereby the tree topology establishment among the clusters towards the base stations in the intercluster communication stage. The overburden node being selected as a gateway node is prevented with the help of an inter-cluster communication procedure. The high-quality clustering algorithm (HQCA) was suggested by Baradaran et al. [9] to make the cluster of high quality. During clustering, the error rates as well as intra and inter clustering distances are enhanced. The fuzzy logic was selected the optimal cluster head $(\mathrm{CH})$ based on different measures including a base station, minimum and maximum distances between the nodes, and residual energy. The node distributions in the network with inter and intracluster intervals evaluate the quality of clusters. When the node distribution is higher means the HQCA chooses several clusters in dense areas.

Table 1: Tabulation based on existing methodologies

\begin{tabular}{|c|c|c|c|c|}
\hline References & Aim of the paper & $\begin{array}{c}\text { Method/Protocol } \\
\text { used }\end{array}$ & Performance measures & Limitations \\
\hline $\begin{array}{l}\text { Karunanithy et } \\
\text { al. [8] }\end{array}$ & $\begin{array}{c}\text { Industrial } \\
\text { automation via IoT and } \\
\text { WSNs }\end{array}$ & CTEEDG & Lifetime and throughput & $\begin{array}{l}\text { Lack of real-time implementation } \\
\text { for industrial applications }\end{array}$ \\
\hline $\begin{array}{l}\text { Baradaran et } \\
\quad \text { al. [9] }\end{array}$ & $\begin{array}{l}\text { Optimal cluster head } \\
\text { selection in WSN }\end{array}$ & HQCA & $\begin{array}{l}\text { Residual energy, } \\
\text { reliability, scalability, } \\
\text { and network lifetime }\end{array}$ & $\begin{array}{l}\text { Needs to include a few } \\
\text { parameters such as the peripheral } \\
\text { density of each node and optimal } \\
\text { cluster estimation for better } \\
\text { efficiency }\end{array}$ \\
\hline $\begin{array}{c}\text { Dhand et al. } \\
{[10]}\end{array}$ & Energy-efficient WSNs & SMEER and SCOR & $\begin{array}{l}\text { Energy consumption } \\
\text { and lifetime }\end{array}$ & Higher computational complexity \\
\hline $\begin{array}{c}\text { Jeske et al. } \\
\text { [11] }\end{array}$ & $\begin{array}{c}\text { Trade-offs among energy } \\
\text { consumption and data } \\
\text { delivery in } \\
\text { large-scale WSNs } \\
\text { determination }\end{array}$ & NSGA-II & $\begin{array}{l}\text { Delivery reliability and } \\
\text { energy consumption } \\
\text { with scalability }\end{array}$ & More time consuming \\
\hline $\begin{array}{c}\text { Stephan et al. } \\
\text { [12] }\end{array}$ & $\begin{array}{l}\text { Interference to primary } \\
\text { users and minimize the } \\
\text { energy consumption of } \\
\text { secondary users }\end{array}$ & SWC-RP and AIIE & $\begin{array}{l}\text { Neighbor connectivity, } \\
\text { Energy, Distance and } \\
\text { Stability }\end{array}$ & Algorithm robustness is poor \\
\hline $\begin{array}{l}\text { Nivedhitha et } \\
\text { al. [13] }\end{array}$ & $\begin{array}{l}\text { Balancing the path } \\
\text { reliability and energy } \\
\text { efficiency in WSNs }\end{array}$ & DMEERP & $\begin{array}{l}\text { Energy consumption, } \\
\text { reliability, network } \\
\text { lifetime, delay, and } \\
\text { packet delivery ratio }\end{array}$ & $\begin{array}{l}\text { Lower performances in case of } \\
\text { data security and energy balance } \\
\text { in the network }\end{array}$ \\
\hline $\begin{array}{l}\text { Seyyedabbasi } \\
\text { et al. [14] }\end{array}$ & $\begin{array}{c}\text { Decentralized IoT } \\
\text { systems and multi-agent } \\
\text { pathfinding in real-time } \\
\text { WSN }\end{array}$ & MAP-ACO & $\begin{array}{l}\text { Distance, Traffic rate, } \\
\text { buffer size, and energy }\end{array}$ & $\begin{array}{l}\text { Higher computational complexity } \\
\text { and more time consuming }\end{array}$ \\
\hline Devi et al. [15] & $\begin{array}{l}\text { Packet loss and latency } \\
\text { reduction in WSNs }\end{array}$ & $\begin{array}{l}\text { Cluster-based data } \\
\text { aggregation scheme }\end{array}$ & $\begin{array}{l}\text { Residual } \\
\text { energy, packet delivery } \\
\text { ratio, overhead, and } \\
\text { latency }\end{array}$ & Higher network delay \\
\hline
\end{tabular}




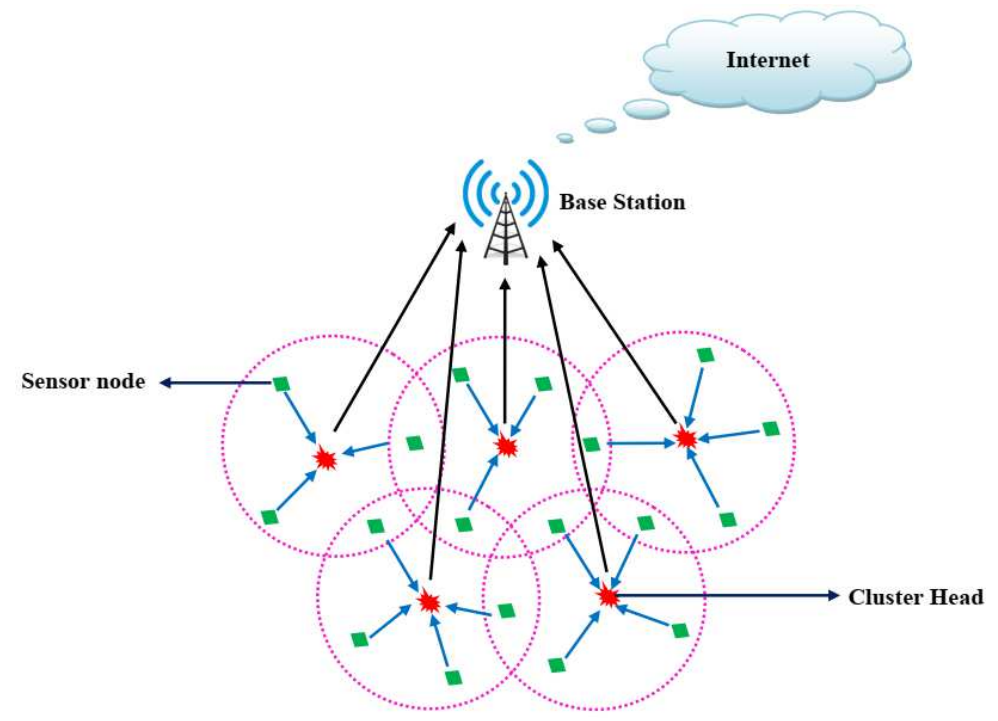

Fig 1: Cluster-based WSN

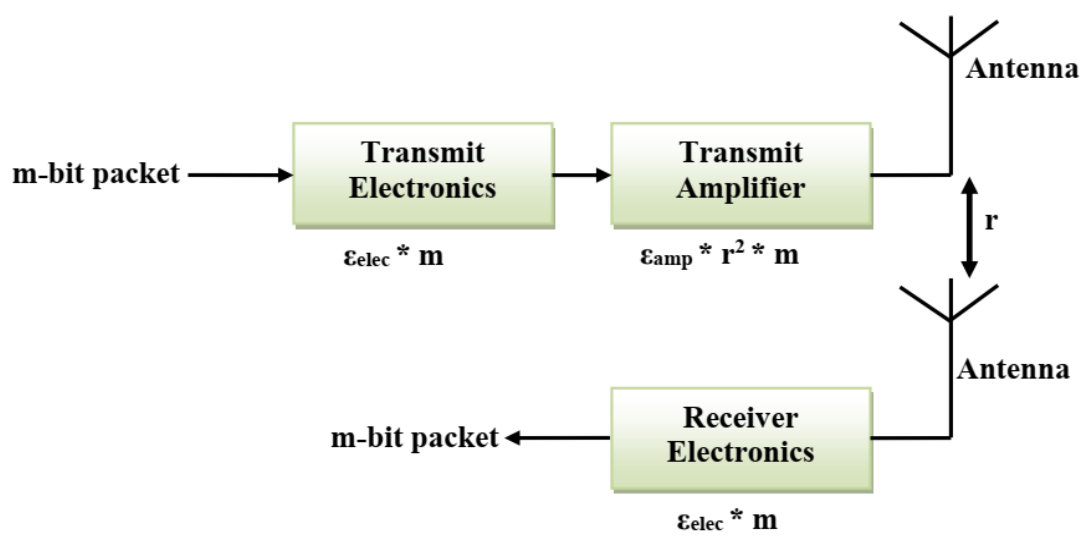

Fig 2: First order radio energy model

Secure Multitier Energy Efficient Routing Protocol (SMEER) and Secure Elliptic curve based Chaotic key Galois cryptography on Opportunistic Routing (SCOR) protocols were introduced by Dhand et al. [10] for optimal energy efficiency in Wireless Sensor Networks. Due to constrained sensor nodes battery limit, the energy efficiency in the major problems in WSNs in which the most relevant cluster head is selected via Ant Lion optimizer with Secure Multi-Tier Energy Efficient Routing Protocol. Jeske et al. [11] proposed the Non-dominated Sorting Genetic Algorithm II (NSGA-II) for both delivery reliability and energy consumption optimization. Based on real case studies, large-scale and medium-sized experimental studies were handled to estimate the performances of delivery reliability as well as energy consumption with better scalability. Because of the structure of their search space, the heuristic model approaches the routing problems.

The spectrum-aware cluster-based routing protocol (SWC-RP) and artificial intelligence-inspired energy (AIIE) were proposed by Stephan et al. [12]. The routing and clustering problems in Cognitive Radio Sensor Network (CRSN) is resolved by using spectrum and energy-aware unequal cluster-based routing (SEUCR) protocol. The CHS selection and rotation minimizes the premature death of $\mathrm{CHs}$ in accordance with neighbor connectivity, distance, energy, and channel stability. The dynamic Multi-hop Energy Efficient Routing Protocol (DMEERP) was established by Nivedhitha et al. [13] to balance the path reliability and energy efficiency in WSNs. All cluster members and $\mathrm{CH}$ records are maintained and stored by using the SuperCluster Head (SCH). If existing fails, the new cluster head estimates the weight factor and node activation. The energy consumption, reliability, network lifetime, delay, and packet delivery ratio performances are evaluated in the network simulator (NS 2.33).

Seyyedabbasi et al. [14] proposed a multi-agent pathfinding-based ant colony optimization (MAP-ACO) algorithm to manage the network resources. In parallel and concurrent conditions, the network lifetime improvement, low energy consumption with the optimal path is determined. While choosing the next destination under different conditions, few parameter performances including distance, traffic rate, buffer size, and remaining energy are evaluated. The packet loss and latency reduction in WSNs are effectively carried out via a cluster-based data aggregation scheme (CDAS), which was proposed by Devi et al. [15]. Particularly, 
Slot scheduling and Aggregation Tree Construction are plays a vital role in C-DAS. The minimum spanning tree (MST) with sink constructs the aggregation tree. The experimental analysis examined higher residual energy and packet delivery ratio with minimum overhead and latency. The survey of existing methodologies is described in Table 1 .

\section{Proposed Methodology}

The aim of the proposed methodology is to attain an energyefficient WSN in terms of both energy and distance. Since the predominant requirement of the WSN is effective data transmission through precise routing to the BS, the proposed method creates an optimum path from the source node to the BS by considering the optimal path and the optimal energy consumption scheme. Hence the proposed method follows two models: The network model and the Radio energy model. The Network model is exploited to form the cluster, and exact Cluster Head selection. The cluster head selection is performed by the modified Brain Storm Optimization Algorithm which comprises BSO and TLBO algorithm. Meanwhile, the Radio Energy model is exploited to analyze energy consumption while transferring and receiving the data.

\subsection{Network Model}

The network model can be established by considering the following protocols. Fig 1 represents the basic structure of WSN.

D Basically, all nodes exhibit the same capabilities with varying energies and are static after the installation

$>$ The nodes may be homogenous or heterogenous

$>$ There is no installation of GPS in nodes and hence the location identification of nodes is not easy

$>$ After the implementation of sensor nodes recharging of the battery is impossible

$>$ The base station is static with a constant power supply

$>$ Each node performs data aggregation i.e., compressing multiple data into a single data packet

$>$ The distance lies between the sensor nodes can be estimated by using Euclidean distance

$>$ The wireless radio link between two nodes is symmetrical i.e., the energy dissipation of data communication from node 1 to node 2 is similar to the data transfer from node 2 to node 1

$>$ The node maintenance is fully performed by the base station and they are implemented arbitrarily in the network area.

$>$ Once a node is dead, its ID cannot be reused for further processing

$>$ BS collects the information from the sensor nodes about the unconsumed energy as well as the distance. This can be further used for the $\mathrm{CH}$ selection incorporated with the modified BSO algorithm.
The energy consumption is more in wireless sensor networks due to several energy-draining activities. In our proposed method the energy consumption is estimated by considering the first-order radio model. Fig 2 depicts the first-order energy model for each sensor node. The energy consumed for the transmission and receiving the data are numerically expressed as below,

Energy consumption at the transmitter side,

$\varepsilon_{T x}(m, r)= \begin{cases}m \times \varepsilon_{\text {elec }}+m \times C_{f s} \times r^{2} & \text { if } r \leq r_{0} \\ m \times \varepsilon_{\text {elec }}+m \times C_{m p} \times r^{2} & \text { if } r>r_{0}\end{cases}$

Energy consumption at the receiver side,

$\varepsilon_{R x}=m \times \varepsilon_{\text {elec }}$

Where $\varepsilon_{\text {Txelec }}$ represents the amount of energy dissipated per bit at the transmitter side and $\varepsilon_{\text {Rx elec }}$ indicates the energy dissipated per bit at the receiver side. Moreover, $r$ represents the interval between the sensor node and its corresponding $\mathrm{CH}, \varepsilon_{\text {elec }}$ estimates the cost of circuit energy while transmitting or receiving one bit of data, $r_{0}$, is the threshold distance, and can be estimated by equation (3).

$r_{0}=\sqrt{\frac{C_{f s}}{C_{m p}}}$

Here, $C_{f s}$ and $C_{m p}$ represent the free space and multipath coefficients of the amplifier respectively. These coefficients completely depend on the amplifier used on the transmitter side. Consider that the distance between the sensor node and its corresponding $\mathrm{CH}(r)$ is less than the threshold value $r_{0}$, then the free space energy model is applied and if it is greater than the $r_{0}$, the multi-path energy model is used. Further, our model exploits the infinite compressibility method to compress the accumulated data in $\mathrm{CH}$ from its sensor nodes to a single packet of fixed length.

\subsection{BrainStorm Optimization Algorithm}

The brainstorm optimization algorithm is ananalytical algorithm developed by yuhui Shi in [16] mainly focused to reduce the issues that occur in multi-modal networks. This is mainly designed in such a way to reduce the real problems [19] in this universe. This algorithm exploits the brainstorming process [17], which is used to form a group of people from different backgrounds in order to generate creative ideas. The brainstorming process follows four rules,

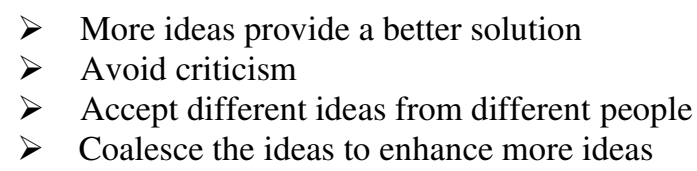

By following the above norms following steps are generated for the selection $\mathrm{CH}$.

i. People from different background are grouped together

\subsection{Radio Energy Model}


ii. Produce a maximum number of ideas corresponding to the above norms

iii. Analyze the problem to select the best ideas

iv. The selected ideas are encounter to generate new ideas

v. From the set of new proposals choose the precise ideas in terms of the problems.

vi. From all these, the best idea is adopted by the problem holder

By considering all the facts the algorithm for BSO is enclosed in algorithm 1.

Algorithm 1 BSO Algorithm

Input: $\mathrm{NP}, \mathrm{L}, \mathrm{P}_{\mathrm{R}}, \mathrm{P}_{1}, \mathrm{P}_{\text {centrel }}, \mathrm{P}_{\text {centre2 }}$

Output: Ideal Clarification of the set at the end of the run

Select NP ideas arbitrarily

While not halt condition do

Grouping operator (NP, L)

If $\operatorname{rand}(0,1)<\mathrm{P}_{\mathrm{R}}$ then

Arbitrarily choose a cluster $\mathrm{C}_{1}$

$\mathrm{B}_{\mathrm{m}}=$ Ideal solution in $\mathrm{C}_{1}$

Replacing operator $\left(\mathrm{B}_{\mathrm{m}}\right)$

end

for $m=1$ to NP do

$\mathrm{X}_{\mathrm{m}}=$ present idea

if rand $(0,1)<\mathrm{P}_{1}$ then

arbitrarily select a cluster $\mathrm{C}_{1}$

if rand $(0,1)<\mathrm{P}_{\text {centrel }}$ then

$\mathrm{B}_{\mathrm{m}}=$ Ideal idea in $\mathrm{C}_{1}$

$\mathrm{Y}_{\mathrm{m}}=$ creating operator $\left(\mathrm{B}_{\mathrm{m}}\right)$

else

$\mathrm{a}_{\mathrm{m}}=$ random idea from $\mathrm{C}_{1}$

$\mathrm{Y}_{\mathrm{m}}=$ creating operator $\left(\mathrm{a}_{\mathrm{m}}\right)$

end

else

arbitrarily chose two clusters $\mathrm{C}_{1}, \mathrm{C}_{2}$

if rand $(0,1)<\mathrm{P}_{\text {centre2 }}$ then

$\mathrm{B}_{\mathrm{m} 1}=$ best idea in $\mathrm{C}_{1}$

$\mathrm{B}_{\mathrm{m} 2}=$ best idea in $\mathrm{C}_{2}$

$\mathrm{C}_{\mathrm{m}}=$ Coalesce $\left(\mathrm{B}_{\mathrm{m} 1}, \mathrm{~B}_{\mathrm{m} 2}\right)$

$\mathrm{Y}_{\mathrm{m}}=$ Creating operator $\left(\mathrm{O}_{\mathrm{m}}\right)$

end

end

select the best idea between $Y_{m}$ and $X_{m}$

end

end

In algorithm $1 N P$ is the number of sensor nodes, $L$ is the total number of clusters, and $P_{R}, P_{l}, P_{\text {centrel }}$, and $P_{\text {centre } 2 \text { are the }}$ probabilities while rand $(0,1)$ send back a real number among $O$ to 1 incorporated with uniform distribution randomly.

In BSO four operators are involved in grouping and are listed below

\section{i. $\quad$ Clustering operators}

The BSO algorithm exploits the k-means algorithm to divide/ cluster the NP quantity of sensor nodes into L clusters. In each cluster, the center point is selected as the best sensor (or) best solution. Hence this operator is used to improvise the search exploration.

\section{ii. Replacing Operator}

The replacing operator is used to replace the center position in the cluster with the best sensor node generated with uniform distribution arbitrarily. This operation is done to avert falling from local optima. The replaced final best sensor node is taken as cluster head.

\section{iii. $\quad$ Creating operators}

From the selected clusters the new idea/solution or cluster head can be generated. These cluster heads are considered as the best centers of the clusters. The new $\mathrm{CH}$ can be generated by appending Gaussian noise to the adopted $\mathrm{CH}$ as shown in equations (4) and (5).

$$
\begin{aligned}
& Y_{m, n}^{i}=X_{m, n}^{i}+\chi_{m, n}^{i} \times N(\mu, \sigma)_{m, n}^{i} \\
& \chi_{m, n}^{i}=\operatorname{rand}(0,1) \times e^{\left(1-\frac{I}{I-i+1}\right)} \times s
\end{aligned}
$$

The total aggregation of iterations is denoted as $I$ and $i$ represents the current iteration. The new idea or cluster head is denoted as $Y_{m, n}^{i}, X_{m, n}^{i}$ is used to denote the randomly selected idea or cluster head of decision variable $n$ of ideas $m$ at iteration $i . N(\mu, \sigma)$ is a vector of Gaussian numbers with mean $\mu$ and variance $\sigma ; s$ is used to represents the step size and rand $(0,1)$ returns the random value along with the uniform distribution function of values lies between 0 and 1 .

\section{iv. Coalescing the Schemes}

Coalescing of schemes is performed to construct a new single cluster head $X_{m, n}^{i}$ and can be given as,

$$
\begin{aligned}
& X_{m, n}^{i}=\operatorname{rand}(0,1) \times X_{m n 1}^{i}+(1-\operatorname{rand}(0,1)) \times X_{m n 2}^{i} \\
& m=1,2, \ldots \ldots, N P, n=1,2, \ldots \ldots . D \\
& Y_{m, n}^{i}=Y_{m, n}^{i}+\operatorname{rand}(1, W) \times C \times\left(Y_{n}^{\text {gbest }}-Y_{m, n}^{i}\right)
\end{aligned}
$$

Here, $X_{m n 1}^{i}, X_{m n 2}^{i}$ are the adopted $\mathrm{CH}$ from cluster 1 and cluster 2correspondingly. $\mathrm{D}$ is the number of decision variables utilized to adopt the new best $\mathrm{CH}$ from the Sensor nodes. $W$ is the coefficient for deciding the width of the random number. $Y_{n}^{\text {gbest }}$ is used to denote the decision variable $n$ of the best $\mathrm{CH}$ so for among all sensor nodes before generating new $\mathrm{CH}$.

Hence the exploitation of this algorithm provides an optimized solution. However, in order to enhance further, we extend this BSO algorithm with some modification to fine-tune the parameters of BSO and it is explained in the following section.

\subsection{Proposed Modified BSO algorithm for cluster head selection}


The BSO algorithm is further enhanced to obtain a betteroptimized cluster head in the selected sensor nodes in WSN. The procedures for the modified version of BSO [18] are explained below.

\section{a. Clustering phase}

Initially, in the clustering phase, the ideas/ sensor nodes (NP) are selected randomly. Then split these sensor nodes/ideas into several clusters based on the fitness-based grouping method. The algorithm for the division of sensor nodes is mentioned in Algorithm 2. Hence, to generate new $\mathrm{CH}$ we have taken equations (4), (6), and (7) from the BSO algorithm. Further, to update the knowledge of sensor nodes we are using the Teacher-learner optimization algorithm in the upcoming section.

Algorithm 2: Clustering by Fitness-based grouping method

Begin
All ideas are ranked according to the evaluation values
for idea $\mathbf{~ m}$
$l=(m-1) \%(N P \div L)+1$
individual 1 is dispatched to cluster 1
end for
end

( $L$ is the total number of clusters)

\section{b. Teacher Learner Phase}

The teacher-learner phase adopts the teacher-learner optimization algorithm to enhances the effectiveness of the BSO algorithm by fine-tuning the parameters.

Let us consider the vector,

$$
X_{m}^{i}=X_{m, 1}^{i}, X_{m, 2}^{i}, \ldots \ldots \ldots X_{m, L}^{i}
$$

Here, $X_{m}^{i}$ denotes the $\mathrm{m}^{\text {th }}$ node in the $\mathrm{i}^{\text {th }}$ iteration whereas, $X_{m, l}^{i}$ represents the $m^{\text {th }}$ node in the cluster $l$ at $i^{\text {th }}$ iteration. Further, $X_{\text {best }}^{i}$ is selected as the best sensor node in the $i^{\text {th }}$ iteration of the algorithm. Since teachers hold more knowledge than the learners, in the teacher-learner phase [20], the best sensor node is considered as a teacher and other nodes are taken as learners. The knowledge of the best $\mathrm{CH}$ can be exploited to update the $m^{\text {th }}$ node in the $l^{\text {th }}$ cluster as follows,

$\tilde{X}_{m}^{i}=X_{m, l}^{i}+F M_{m, l}$

Where $F M_{m, l}$ can be defined as,

$$
F M_{m, l}=\operatorname{rand}(0,1) \times\left(X_{\text {best }, l}^{i}-D \times M_{l}^{i}\right)
$$

Here, $M_{l}^{i}$ represents the mean nodes in the $l^{\text {th }}$ cluster, D indicates the deciding factor in the estimation of the mean value of nodes $M_{l}^{i}$. It can be computed as follows,

$D=\operatorname{round}(1+\operatorname{rand} \times(2-1))$

The round function in equation (11) is used to round off the calculated value to the nearest integer.
From the updated function $\tilde{X}_{m, l}^{i}$ the performance function $f\left(\tilde{X}_{m}^{i}\right)$ of the learner sensor node $\tilde{X}_{m}$ can be estimated. Then the computed value is compared with the performance of $\tilde{X}_{m}^{i+1}$ and the resultant equation can be given as,

$\tilde{X}_{m}^{i+1}=\left\{\begin{array}{l}\tilde{X}_{m}, \text { if } f\left(\tilde{X}_{m}\right)<f\left(X_{m}^{i}\right) \\ X_{m}^{i} ; \text { otherwise }\end{array}\right.$

Meanwhile, in the learner phase, the learner nodes improvise their comprehension by interlinking with other nodes. The knowledge enhancement of learner nodes totally depends on the subsequent learner. The learning phase can be described as follows,

Let us consider $\mathrm{P}$ and $\mathrm{Q}$ nodes. The node $\mathrm{P}$ can be updated as,

$$
\tilde{X}_{P l}=X_{P, l}^{i}+\operatorname{rand}(0,1) \times\left(X_{P, l}^{i}-X_{Q, l}^{i}\right) \text { if } f\left(X_{P}^{i}\right)<f\left(X_{Q}^{i}\right)
$$

and

$\tilde{X}_{P l}=X_{P, l}^{i}+\operatorname{rand}(0,1) \times\left(X_{Q, l}^{i}-X_{P, l}^{i}\right)$ if $f\left(X_{Q}^{i}\right)<f\left(X_{P}^{i}\right)$

After updating $\tilde{X}_{P}$, the performance function $f\left(\tilde{X}_{P}\right)$ is calculated and hence $\tilde{X}_{P}^{i+1}$ can be obtained as follows,

$\tilde{X}_{P}^{i+1}=\left\{\begin{array}{l}\tilde{X}_{P} ; \text { if } f\left(\tilde{X}_{P}\right)<f\left(\tilde{X}_{P}\right) \\ X_{P}^{i} ; \text { otherwise }\end{array}\right.$

Thus this phase improvises the parameters of the BSO algorithm by updating the knowledge of sensor nodes. Further, to adopt the ideal $\mathrm{CH}$ from the updated nodes we are using the ranking phase as shown below.

\section{c. Ranking Phase}

In the ranking phase, the fitness function of our proposed modified BSO algorithm is exploited to adopt the ideal $\mathrm{CH}$ from the ranked sensor nodes of the selected cluster. Some of the fitness functions are listed below,

\section{- $\quad$ Residual energy}

Residual energy is estimated to ignore the intervention of dead nodes in the $\mathrm{CH}$ adoption process. The residual energy $\mathrm{E}_{\mathrm{R}}$ can be determined as follows,

$$
E_{R}=\sum_{l=1}^{L} \frac{1}{E_{R, C H l}}
$$

Where, $E_{R, C H l}$ is the residual energy of the $l^{\text {th }}$ cluster head. Since $\mathrm{CH}$ requires more energy to perform the function of transmitting data to the BS from the normal sensor nodes, the node which can hold more residual energy can be taken as the best $\mathrm{CH}$.

\section{- $\quad$ Node Degree}

The node degree is predominantly important to rank the $\mathrm{CH}$ in terms ofthe number of sensor nodes it holds. The $\mathrm{CH}$ with less number of normal sensor nodes can conserve more energy for 
future tasks than the $\mathrm{CH}$ with more number of nodes. Hence node degree is exploited to adopt the preferred $\mathrm{CH}$ with less quantity of sensor nodes. Thus it can be denoted by the integer of sensor nodes belong to the corresponding $\mathrm{CH}$ and can be given as,

$$
E_{N}=\sum_{l=1}^{L} N P_{l}
$$

Where $N P_{l}$ is the number of sensor nodes held by $\mathrm{CH}_{l}$.

\section{- Interval among the sensor nodes}

The intervalamong the nodes is estimated to choose the exact $\mathrm{CH}$ in order to minimize energy usage. It can be defined by the interval among the normal nodes and its own $\mathrm{CH}$ in a cluster for the effective transmission of data to BS. Besides, the sensor node's energy dissipation is increase with an increase in transmission distance as mentioned in section 3.2. Hence the interval among the normal nodes and its own $\mathrm{CH}$ can be defined as,

$$
E_{D}=\sum_{b=1}^{L} \sum_{l=1}^{L b} \operatorname{dis}\left(N P_{l}, C H_{b}\right) / L_{b}
$$

Here, the interval between the sensor $l$ and $\mathrm{CH}_{b}$ is determined as $\operatorname{dis}\left(\mathrm{NP}_{l}, \mathrm{CH}_{b}\right)$ and sensor nodes belong to $\mathrm{CH}_{b}$ is determined as $L_{b}$.

\section{- Distance between Cluster head and BS}

Similarly, this function is also exploited to choose the ideal $\mathrm{CH}$ to reduce energy usage by nodes. Basically, energy consumption purely depends on the distance, and hence if the $\mathrm{CH}$ lies far away from the BS, then during transmission of data it requires more energy. Then it can be defined as the interval among the $\mathrm{CH}$ to $\mathrm{BS}$ and can be given as,

$$
E_{B}=\sum_{l=1}^{L} \operatorname{dis}\left(\mathrm{CH}_{b}, B S\right)
$$

Here the interval among the Cluster head $\mathrm{CH}_{b}$ and BS is determined as $\operatorname{dis}\left(\mathrm{CH}_{b}, \mathrm{BS}\right)$.

\section{- $\quad$ Node Centrality}

The node centrality function is used to lessen the transmission distance between the cluster nodes and $\mathrm{CH}$. The higher the node centrality, the more reduction in transmission distance and energy. It can be defined as how $\mathrm{CH}$ is positioned centrally from neighbor nodes and can be expressed as,

$$
E_{C}=\sum_{l=1}^{m} \frac{\sqrt{\sum_{b \in n} \operatorname{dist}^{2}(l, b) / n(l)}}{\text { Network Dimension }}
$$

Here, $n(l)$ is used to represent the adjacent nodes of $\mathrm{CH}_{l}$.

\subsection{Optimal cluster head selection}

The optimal cluster can be selected by evaluating the weighted value of the above-mentioned fitness functions. It can be given as,

$$
E_{1}=\rho_{1} E_{R}+\rho_{2} E_{D}+\rho_{3} E_{B}+\rho_{4} E_{N}+\rho_{5} E_{C}
$$

Here the values of $\rho_{i}$ lies between 0 and 1 and $\sum \rho_{i}=1$. Therefore consider the values of $\rho_{1}, \rho_{2}, \rho_{3}, \rho_{4}$, and $\rho_{5}$ as 0.4 , $0.2,0.15,0.13$, and 0.12 respectively.

The benefits of selecting optimal $\mathrm{CH}$ are given below,

Long-lasting network life

$>$ Reduction in energy consumption for transmitting data from sensor nodes to the BS

$>$ Reliability enhancement: Since the hops between the nodes and BS minimize the packet delivery ratio, the heterogeneous network holds lesser hops than the homogenous network. Hence it accomplishes a better packet delivery ratio than the homogenous network and thus there is an improvement in reliability

$>$ Reduction in Latency: The reduction in the number of hops among the nodes and BS minimizes the forwarding latency

\subsection{Node allocation using a potential function}

After the effective optimal selection of $\mathrm{CH}$ by our proposed modified BSO algorithm, the allocation of sensor nodes to the specific $\mathrm{CH}$ begins by exploiting potential function.

$S N=\frac{z \times \operatorname{Energy}\left(\mathrm{CH}_{b}\right)}{\operatorname{Dist}\left(\mathrm{NP}_{l}, \mathrm{CH}_{b}\right)}$

Here, $S N$ is used to represents the sensor node prospective, and $z$ is the correlation constant; $\operatorname{dist}\left(\mathrm{NP}_{l}, \mathrm{CH}_{b}\right)$ indicates the distance between the cluster head and sensor nodes; energy $\left(\mathrm{CH}_{b}\right)$ denotes the unconsumed energy of the corresponding $\mathrm{CH}$.The nearest $\mathrm{CH}$ with higher potential is allocated to the sensor nodes. For instance, if two or more $\mathrm{CH}$ are located at the same distance from a sensor node, then $\mathrm{CH}$ which holds maximum energy can be allocated as a $\mathrm{CH}$ to it.

\subsection{Energy-efficient data routing}

The CHs transfer the collected data from their respective clusters to the BS. The next important step is the selection of forwarding nodes. The forwarding nodes must exhibit higher residual energy than the other normal nodes. Hence it enhances the packet delivery ratio and prevents the packets from dropping during transmission. By exploiting our proposed modified BSO method, the nodes are selected based on the higher delay to provide efficient node to node transmission.

$E_{L}=\frac{\sum_{l=1}^{S} E_{0}\left(C H_{b, l}\right)}{\sum_{l=1}^{S} E\left(C H_{b, l}\right)}$

Where $S$ is the sum of cluster heads and $E_{L}$ is the leftover energy of a dynamized sensor node, $E_{0}\left(C_{b, l}\right)$ initial nodal energy of $\mathrm{CH}_{l}$ of cluster $b . E\left(\mathrm{CH}_{b, l}\right)$ indicates the unconsumed energy for that specific cluster head.

\subsection{Network coverage}


In the sensing areas, network coverage is determined as the union of all distributed sensor nodes. However, the probability of better coverage of the network can be given as 1 and 0 for all other options.Consider a dynamized sensor node and it transmits the data within the network broadcasting. The interval among the energized sensor node and the cluster head can be estimated by using the Euclidean distance $D=\left(a_{1}-a_{2}\right)^{2}+\left(b_{1}-b_{2}\right)^{2}$ and it can be given as below,

$$
C_{a, b}=\left\{\begin{array}{l}
\text { if } \sqrt{\left(a_{1}-a_{2}\right)^{2}+\left(b_{1}-b_{2}\right)^{2}} \leq R \\
\text { 0if } \sqrt{\left(a_{1}-a_{2}\right)^{2}+\left(b_{1}-b_{2}\right)^{2}} \geq R
\end{array}\right.
$$

Here, we are considering the binary sensing model, and $\mathrm{a}, \mathrm{b}$ are the coordinates of the sensing node, $R$ sensing coverage range, $C_{a, b}$ is the coverage. The effectiveness of the network coverage can be analyzed by the scalability of the routing protocol. However, the scalability enhances with the expansion ofthe number of nodes in a cluster. Hence scalability can be defined as the effective functioning of a network with the massive enhancement of network size. Besides, the size of the clusters can be increased by adding unclusters nodes with the help of routing protocol.

$$
C=\frac{N P^{e}}{\sum_{l=1}^{L} C^{l}}-1
$$

The total number of all dynamized sensor nodes in a grid can be given as $N P^{e}$.

\subsection{Transmission quality Ratio}

The transmission quality of the network can be analyzed by estimating RSSI (Received Signal Strength Indicator). RSSI is exploited to enhance node pair communication and notifies the quality of the network. It is the registered standard in the CC2420 transceiver to estimate the network functioning on the receiver side [22]. The signal strength may weaken with the increase in distance and hence the transmission quality decreases which impacts the data throughput [21]. Therefore to estimate the transmission quality between the sensor node and $\mathrm{CH}$ the following equation is used.

$T_{b}=\frac{R_{b}\left(N P_{b}, C H_{b, l}\right)}{\min R_{b}}$

Where $R_{b}\left(N P_{b}, C H_{l, b}\right)$ indicates the RSSI for the transmission correlation from an individual node $N P_{b}$ to the cluster head $\mathrm{CH}_{b, l} . \min R_{b}$ indicates the inferior RSSI value for all transmission links. The transmission quality decreases with increasing $T_{b}$ value [22]. Hence, the fitness function utilized for the estimation of an energy-efficient Routing protocol can be given as,

$$
E_{2}=\kappa_{1} E_{L}+\kappa_{2} C+\kappa_{3} T_{b}
$$

Where $\kappa_{1}, \kappa_{2}, \kappa_{3}$ are the weight coefficients that are used for each underactive in the chief fitness function. i.e., the summation of all three coefficients is equal to 1 . The overall functions of the proposed methodology are illustrated in the flow chart below.

Figure 3 shows that the optimal $\mathrm{CH}$ selection carried out by our proposed BSO-TLBO algorithm by considering performance metrics such as Residual energy, Node degree, the intervalamong the sensor nodes, the interval among the sensor node and $\mathrm{CH}$, and Node centrality for the effective transmission of data along with the sensor nodes and BS. The frequent monitoring of residual energy by BS can avert node failures. Therefore our proposed method can be utilized to design an energy-efficient WSN in order to enhance the grid lifetime and to attain a transmission quality rate.

\section{Simulation and outcomes}

To analyze the performance of our proposed method BSOTLBO based algorithm for the Energy-efficient weighted clustering method for WSN is compared with recently presented methods known as WOA based EECH method [23], BSO based EECH method [24], BOA-ACO based EECH method [25].

\subsection{Simulation Environment}

The simulation of all the existing and our proposed method is implemented in MATLAB R2018a which is operated in windows 8 OS with an Intel Core i3 processor and $6 \mathrm{~GB}$ RAM. To obtain the effectiveness of the different algorithms along with the proposed method, we have executed and checked with 27 different network topologies. The simulation criteria are enclosed in table 2. Extensive simulations are conducted by considering different scenarios, varying the node density, and starting energy.

The position of BS has a great impact on different energy parameters like energy consumption, transmission quality ratio, etc., To analyze the impact we have considered 3 different scenarios. Outline 1: BS is located at the center of the network.Outline 2: BS is located at the edge of the grid. Outline 3: BS is located away from the grid. These are illustrated in figures 4 (a), (b), and (c) respectively. The simulation parameters utilized for our proposed method is tabulated in table 2 .

Table 2: Simulation criteria

\begin{tabular}{|l|l|}
\hline Network Area & $350 \mathrm{X} 350$ \\
\hline Starting Nodal Energy, $\mathrm{E}_{0}$ & $0.4 \mathrm{~J}$ \\
\hline $\begin{array}{l}\text { Total nodes present in a } \\
\text { system }\end{array}$ & $100-250$ \\
\hline $\begin{array}{l}\text { The energy dissipated per bit, } \\
\varepsilon_{\text {elec }}\end{array}$ & $50 \mathrm{~nJ} / \mathrm{bit}$ \\
\hline Message size amplifying & $4500 \mathrm{bits}$ \\
\hline $\begin{array}{l}\text { Transmitter } \\
\text { multi-path coefficient, } \mathrm{C}_{\mathrm{mp}}\end{array}$ & $0.001310 \mathrm{pJ} / \mathrm{bit}^{\mathrm{m}} / \mathrm{m}^{4}$ \\
\hline $\begin{array}{l}\text { Transmitter amplifying free } \\
\text { space coefficient, } \mathrm{C}_{\mathrm{fs}}\end{array}$ & $10 \mathrm{pJ} / \mathrm{bit} / \mathrm{m}^{2}$ \\
\hline Propagation Model & $\begin{array}{l}\text { Free space and Multipath } \\
\text { fading }\end{array}$ \\
\hline
\end{tabular}




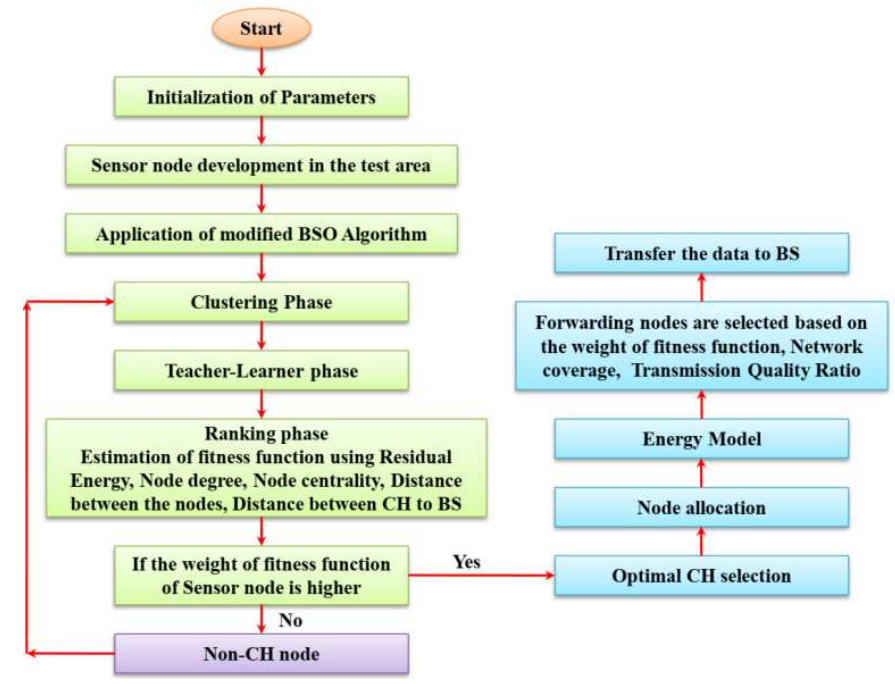

Fig 3: Flow diagram of the proposed methodology
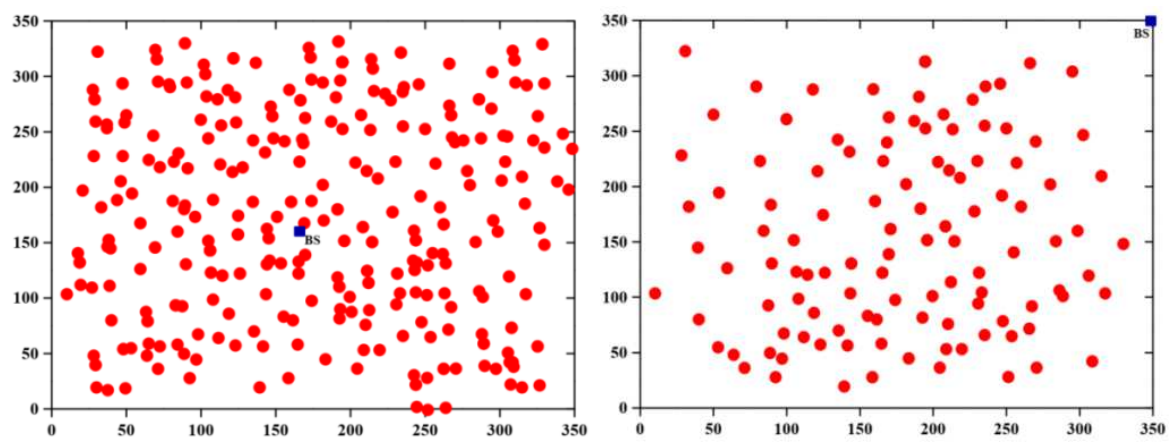

(a)

(b)

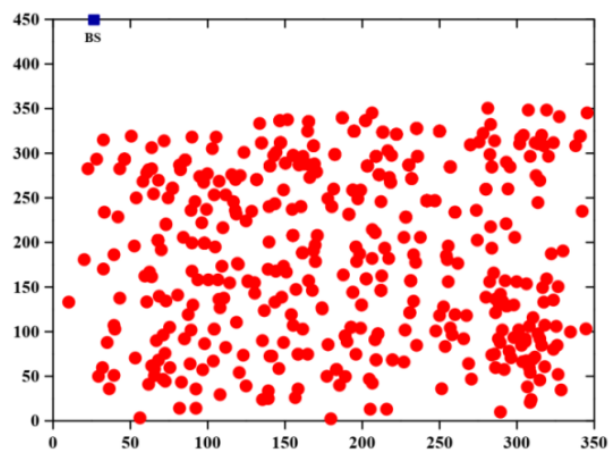

(c)

Fig 4: (a) Outline 1, (b) Outline 2, (c) Outline 3

\subsection{Performance analysis}

Evaluation of cluster-based routing is performed by considering different performance metrics such as FND, HND, LND, dead nodes, active nodes, Average energy consumption, network lifetime,

First Node Dies (FND): FND determines the total aggregations of runs completed before the initial node of the simulated networks dies.
Half Nodes Dies (HND): HND determines the total aggregations of runs completed before $50 \%$ of nodes of the simulated networks die.

Last Node Dies (LND): LND determines the total aggregations of runs completed when all the nodes in the simulation networks die.

Grid Lifetime: The lifetime of the network can be determined by considering three metrics, FND, HND, LND.

Number of Packets received at BS: This is used to determine the number of packets transmitted by the network to the BS. 
The maximum number of packets reached the destination i.e., the BS the lower death rate of the nodes and energy consumption [26].

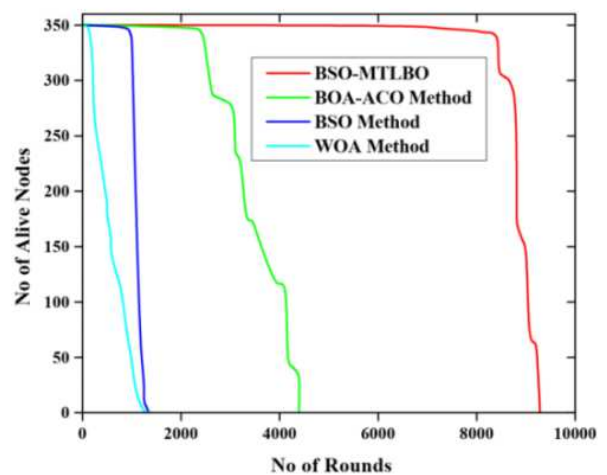

Fig 5: Performance analysis based on active nodes for outline 1

Mean energy consumption: The meanquantity of energy consumed during each round in the simulation networks is estimated by this parameter.

Active nodes: Active nodes determine the performance ability of the network. Higher the alive nodes better the network performance.

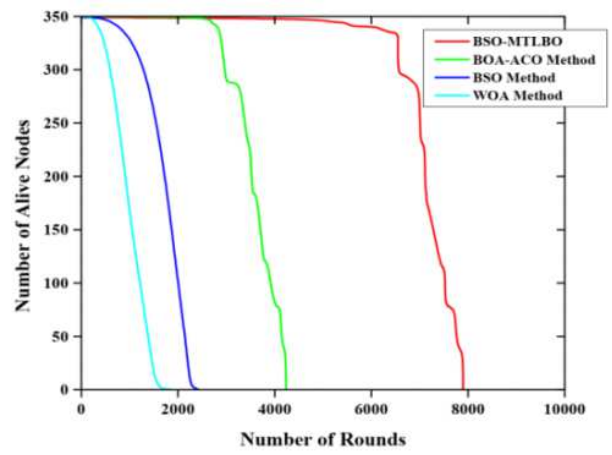

Fig 6: Performance analysis based on active nodes for outline 2

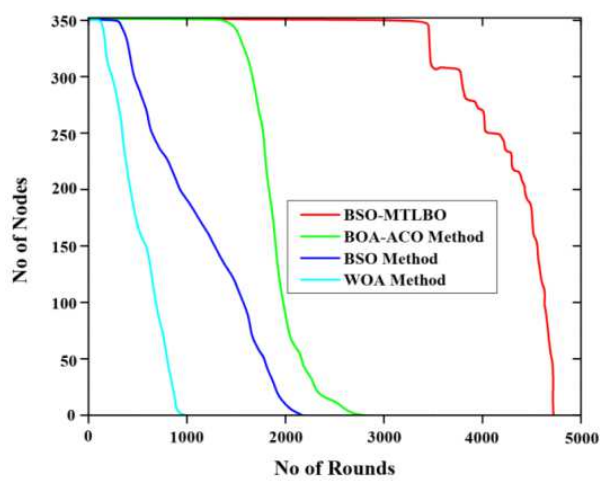

Fig 7: Performance analysis based on active nodes for outline 3

Routing overhead: This occurs due to the replica of data in the routing path and can be determined as the proportion of the total number of packets received by the BS and the total number of transmitted packets.

Throughput: It can be determined as the number of transmitted packets to the Base Station through sensor nodes. It is measured as bits per sec.

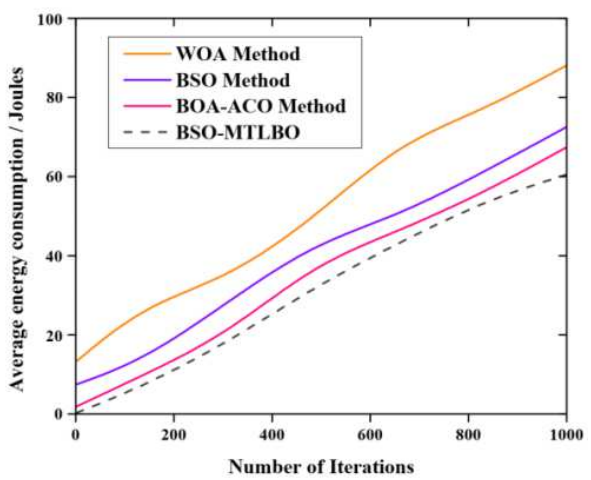

Fig 8: Performance analysis based on mean energy consumption for Outline 1

\subsection{Performance analysis based on active nodes}

The performance analysis in terms of active nodes are conducted for our proposed work with the existing WOA based EECH method [23], BSO based EECH method [24], and BOA-ACO based EECH method [25] works. The simulations are carried for 350 sensor nodes at three above mentioned scenarios and are illustrated in Fig 5, 6, and 7. Fig 5 depicts that our proposed methodology performs better than the existing works for scenario 1 and persists up to almost 9500 iterations, whereas, BOA-ACO method persists for 4200 iterations, and BSO and WOA methods persist only up to 1800 iterations. The performance-based on alive nodes for scenario 2 is better than the other works as shown in fig 6 . Fig 6 depicts that in our proposed method the nodes withstand until 7000 iterations and the BOA-ACO method withstand until approximately around 4000 iterations. The least performances are obtained from the other two methods. For outline 3 also our method outperforms other works as illustrated in fig 7 . In this position also nodes of our proposed method withstand until 3200 iterations and started node failures after that.

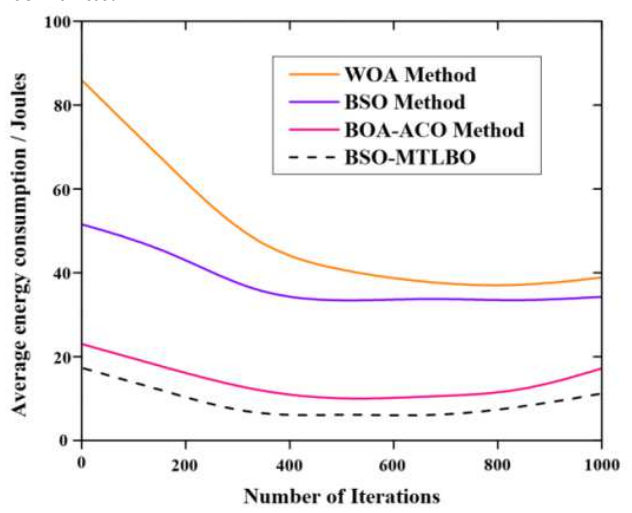

Fig 9: Performance analysis based on mean energy consumption for outline 2 


\subsection{Performance analysis in terms of mean energy consumption}

The performance analyses based on average energy consumption for scenarios 1, 2, and 3 are illustrated in Figures 8,9 , and 10. For each scenario, our proposed BSO-MTLBO algorithm outperforms the existing works.

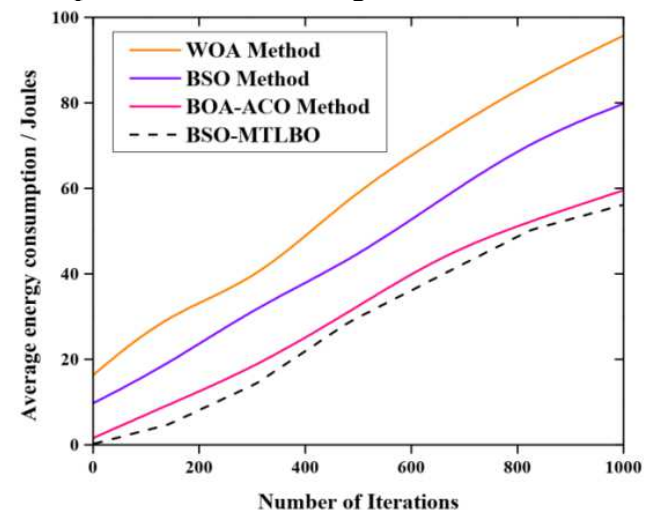

Fig 10: Performance analysis based on mean energy consumption for outline 3

\subsection{Performance analysis based on grid lifetime:}

The network lifetime can be determined with the aid of coverage ratio and the maximum coverage ratio denotes the secured and powerful network lifetime. The performance analysis of coverage ratio against the aggregated executions and number of transmitting nodes is illustrated in figures 11 and 12 respectively. Our proposed work follows optimized $\mathrm{CH}$ grouping after the formulation of clustering and hence our method outperforms the other approaches and provides a better network lifetime as shown in figures 11 and 12 . The coverage ratio of our proposed work is greater than $92 \%$ at iteration 1000 and for BOA-ACO it is around $76 \%$ and for BSO it is around $62 \%$ whereas, WOA exhibits $34 \%$ as shown in fig 11. While comparing the coverage ratio with the number of nodes, our proposed method achieves 96\%, BOA-ACO achieves $88 \%$, BSO accomplishes $70 \%$, and WOA accomplishes $57 \%$ of the coverage ratio for 350 number of sensor nodes as shown in fig 12 .

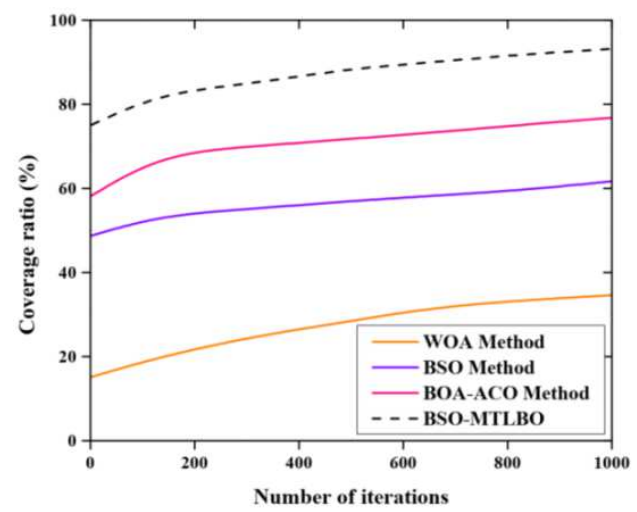

Fig 11: Performance analysis of Network lifetime (coverage ratio vs Number of iterations)

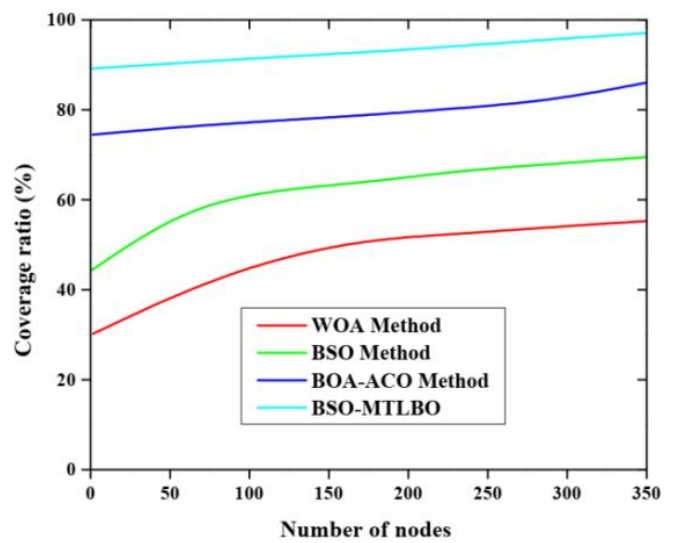

Fig 12: Performance analysis of Network lifetime (coverage ratio vs Number of nodes)

\subsection{Performance analysis based on Dead sensor nodes}

To analyze the performance of our proposed method based on dead nodes, consider the metrics FND, HND, and LND. For all metrics, our proposed method shows better results than the existing approaches for each scenario as shown in figures 13 , 14 , and 15 .

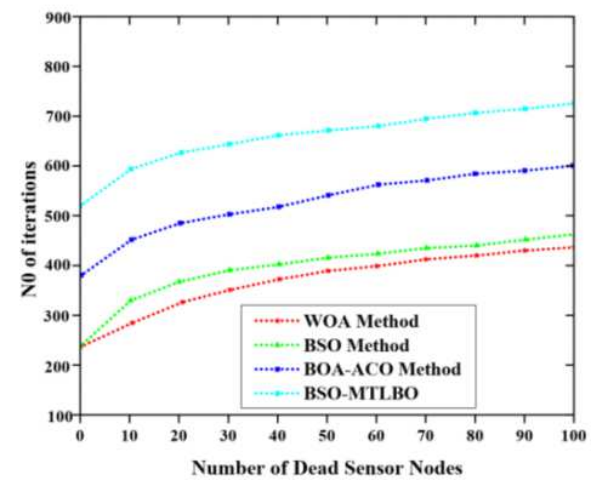

Fig 13:Performance analysis based on Dead sensor nodes for outline 1

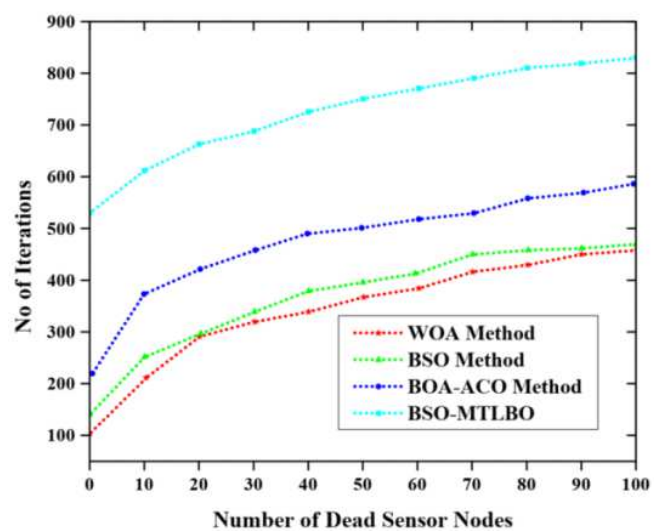

Fig 14: Performance analysis based on Dead sensor nodes for outline 2 


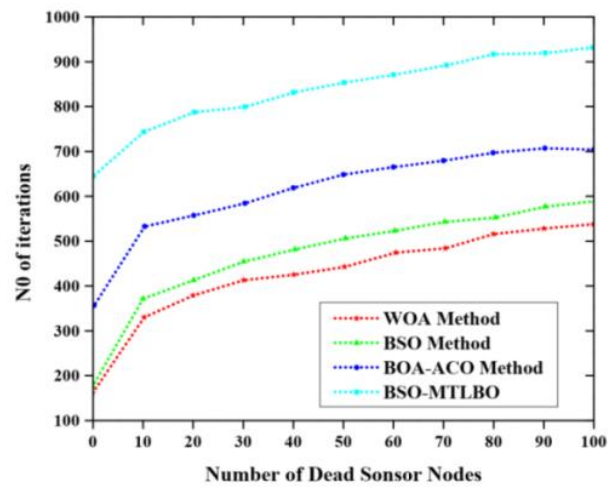

Fig 15: Performance analysis based on Dead sensor nodes for outline 3

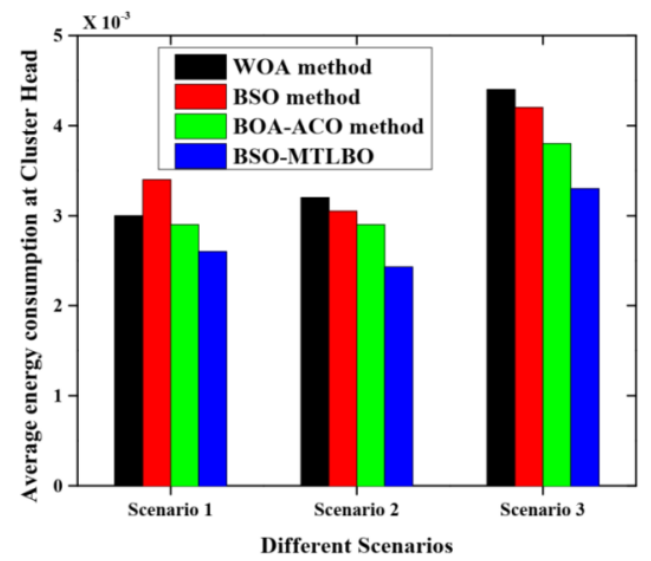

Fig 16:Performance analysis of Average energy consumption at the Cluster head

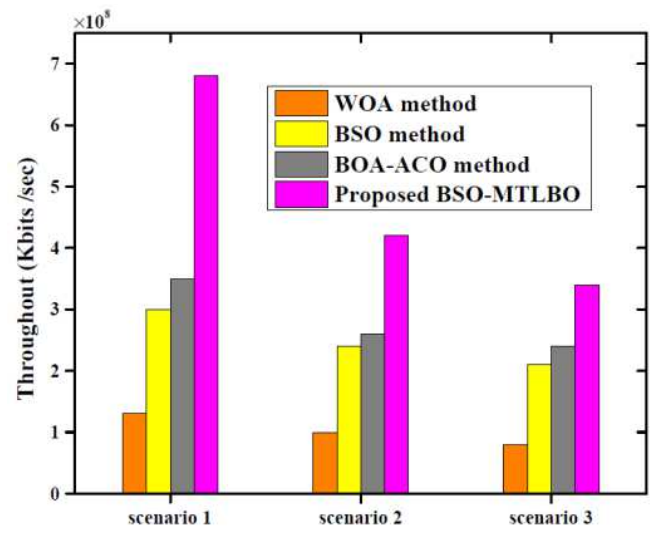

Fig 17: Performance analysis based on throughput for scenarios 1,2 , and 3

\subsection{Performance analysis based on average energy consumption at Cluster Head}

The simulation results of performance analysis based on average energy consumption at cluster head are shown in fig 16. Our proposed work enhances a novel fitness function to rank the nodes in the cluster and hence it reduces the energy consumption at $\mathrm{CH}$, whereas the existing work follows an uneven load between the clusters. Thus our proposed work consumes less energy than the other existing approaches as shown in fig 16.

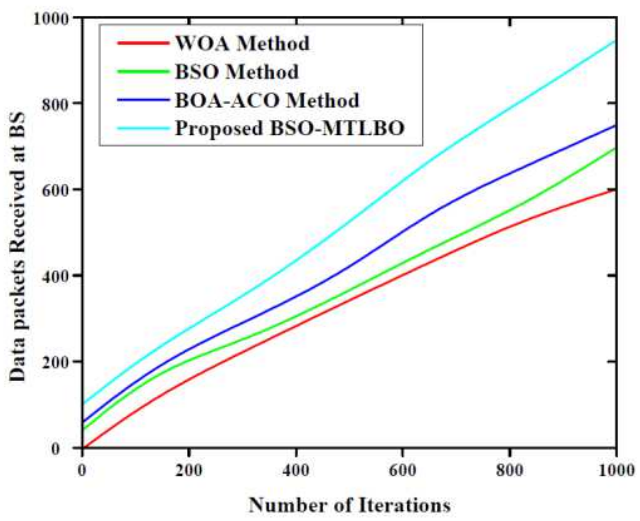

Fig 18: Performance analysis based on the data packet derived against the aggregated runs

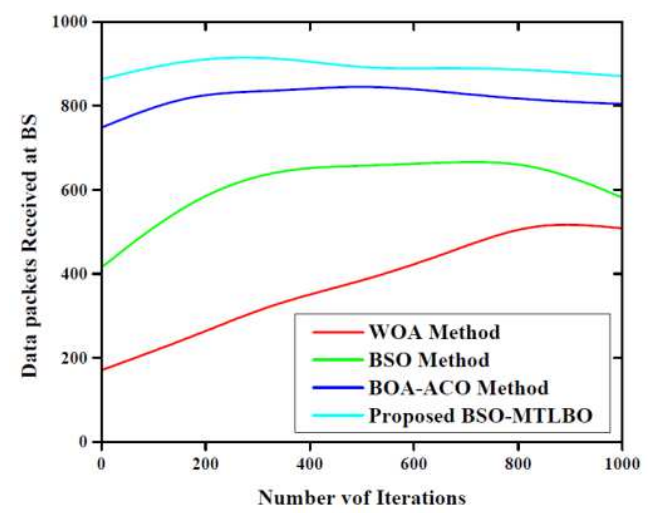

Fig 19: Performance analysis based on the data packet derived against the aggregated nodes

\subsection{Performance analysis based on throughput}

The simulation outcomes of performance analysis based on throughput are shown in fig 17, for different scenarios. The throughput of the system generally depends on two factors: less energy consumption and the maximum aggregated data packets dispatched to the BS. The comparative study shows that our proposed method has better throughput than the existing approaches since our method follows optimal cluster formulation and selection of $\mathrm{CH}$. Due to this reason, the energy consumption got reduced and hence it increases the throughput. Moreover, it also permits to transmission higher number of data to BS, thus the throughput also increases. The higher throughput shows our proposed method has better transmission quality of ratio.

\subsection{Performance analysis based on the data packet received and routing overhead}

The performance analysis based on the data packet received is shown in Figures 18 and 19. The higher the data packets received better the performance is. Our proposed method transmits more data packets to BS due to the optimal route 
selection provided by energy-efficient weighted based clustering. The fitness functions used in the ranking phase offers better results than the other approaches.

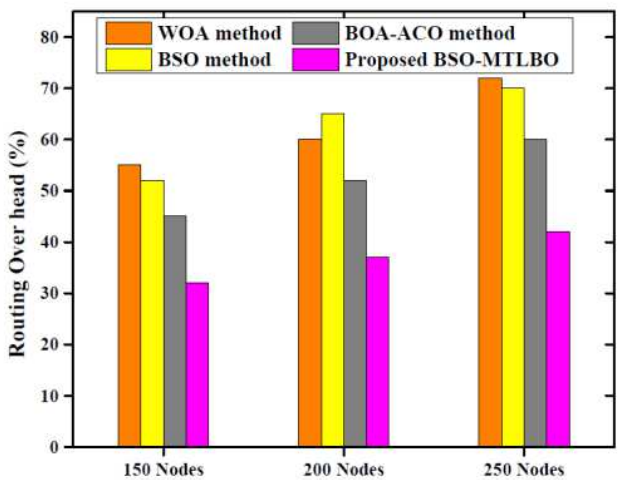

Fig 20: Performance analysis based on routing overhead for different nodes

Moreover, fig 20 depicts the performance analysis of our proposed system based on routing overhead. Since our method provides higher throughput and transmits more data to the BS, the routing overhead is less when compared to other approaches. Fig 20 shows the performance analysis based on routing overhead for a various number of nodes. Thus BSO based TLBO algorithm provides better performance than the other recent approaches.

\section{Conclusion}

The efficacy of the WSN can be estimated with optimal $\mathrm{CH}$ selection and routing. To achieve this requirement we exploited a modified BSO algorithm which combines the BSO algorithm and teacher-learner optimized algorithm. The teacher-learner optimized algorithm is used to fine-tune the parameters of the BSO algorithm. The ranking phase utilizes five fitness parameters to select the clustered sensor nodes and cluster head. The optimal routing was selected to reduce the energy consumption at $\mathrm{CH}$ as well as to prevent packet loss and node failures. Further, the performances were analyzed by considering three scenarios based on the location of the base station. The comparative study for different performance metrics shows that our proposed work outperforms all the existing works we have taken. The network lifetime also got improved when compared to other approaches. In the future, the routing algorithm can be improved by considering different fitness functions. Also, the search speed can be improved by using different hybrid optimization techniques.

\section{COMPLIANCE WITH ETHICAL STANDARDS}

\section{Conflict of interest}

The authors declare that they have no conflict of interest.

\section{Human and Animal Rights}

This article does not contain any studies with human or animal subjects performed by any of the authors.

\section{Informed Consent}

Informed consent was obtained from all individual participants included in the study.

\section{References:}

1. Sankaralingam, S.K, Nagarajan, N.S, \& Narmadha, A.S. (2020) Energy aware decision stump linear programming boosting node classification based data aggregation in WSN. Computer Communications.

2. Maheshwari, K, Prachi, Ajay. Sharma, and Karan Verma. (2020) Energy efficient cluster based routing protocol for WSN using butterfly optimization algorithm and ant colony optimization. Ad Hoc Networks 110, 102317.

3. Singh, Ramnik, and Anil Kumar Verma. (2017) Energy efficient cross layer based adaptive threshold routing protocol for WSN. AEU-International Journal of Electronics and Communications 72, 166-173.

4. Sood, Tanvi. (2020) LUET: A novel Lines-of-Uniformity based Clustering protocol for Heterogeneous-WSN for multiple-applications. Journal of King Saud UniversityComputer and Information Sciences.

5. Singh, A. \& Nagaraju, A. (2020) Low latency and energy efficient routing-aware network coding-based data transmission in multi-hop and multi-sink WSN. Ad Hoc Networks, 107, 102182.

6. Sharma, Neelam, B.M, Singh, and Karan Singh. (2020) QoS-Based Energy-Efficient Protocols for Wireless Sensor Network. Sustainable Computing: Informatics and Systems 100425.

7. Samara, Ghassan, and Mohammad Aljaidi. (2019) Efficient energy, cost reduction, and QoS based routing protocol for wireless sensor networks. arXiv preprint arXiv, 1903.09636.

8. Karunanithy, Kalaivanan, and Bhanumathi Velusamy. (2020) Cluster-Tree based Energy Efficient Data Gathering Protocol for Industrial Automation using WSNs and IoT. Journal of Industrial Information Integration, 100156.

9. Baradaran, A.A, \& Navi, K. (2020) HQCA-WSN: Highquality clustering algorithm and optimal cluster head selection using fuzzy logic in wireless sensor networks. Fuzzy Sets and Systems, 389 114-144.

10. Dhand, G, \& Sheoran, K. (2020) Protocols SMEER (Secure Multitier Energy Efficient Routing Protocol) and SCOR (Secure Elliptic curve based Chaotic key Galois Cryptography on Opportunistic Routing). Materials Today: Proceedings.

11. Jeske, Marlon, Valério Rosset, and Mariá CV Nascimento. (2020) Determining the Trade-offs Between Data Delivery and Energy Consumption in Large-scale WSNs by Multi-Objective Evolutionary Optimization. Computer Networks 107347.

12. Stephan, Thompson, Fadi Al-Turjman, Suresh Joseph, K. (2020) Balamurugan Balusamy, and Sweta Srivastava. 
Artificial intelligence inspired energy and spectrum aware cluster based routing protocol for cognitive radio sensor networks. Journal of Parallel and Distributed Computing.

13. Nivedhitha, V, Gopi Saminathan, A, and Thirumurugan, P. (2020) DMEERP: A dynamic multi-hop energy efficient routing protocol for WSN. Microprocessors and Microsystems 79103291.

14. Seyyedabbasi, Amir, and Farzad Kiani. (2020) MAPACO: An Efficient Protocol for Multi-Agent Pathfinding in Real-Time WSN and Decentralized IoT Systems. Microprocessors and Microsystems 103325.

15. Devi, Seedha, V, Ravi, T, and Baghavathi Priya. S. (2020) Cluster Based Data Aggregation Scheme for Latency and Packet Loss Reduction in WSN. Computer Communications 149 36-43.

16. Shi, Y. (2011) Brainstorm optimization algorithm. In: Proc. 2nd Int. conf. on swarm intelligence, 303-309.

17. Osborn, A. F. (1957) Applied Imagination, New York: Scribner.

18. Arai, Kiyo, Yoshikazu Fukuyama, Tatsuya Iizaka, and Tetsuro Matsui. (2019) Optimal Operational Planning of Energy Plants Considering Uncertainty of Renewable Energy Outputs by Global-best Brain Storm Optimization. IFAC-PapersOnLine 52 (4) 153-158.

19. Shi, Y. (2015) Brain Storm Optimization Algorithm in Objective Space. Proc. of IEEE CEC.

20. Rao, R.V, Savsani, V.J, Vakharia, D.P. (2011) Teaching-learning-based optimization: a novel method for constrained mechanical design optimization problems, Comput. Aided Des. 43 (3) 303-315.
21. Ansere, J. A, Han, G, and Wang, H. (2019) A Novel Reliable Adaptive Beacon Time Synchronization Algorithm for Large-Scale Vehicular Ad hoc Networks, IEEE Transactions on Vehicular Technology. doi: 10.1109/TVT..2946225.

22. Ansere, J. A, Bonsu, K. A, Abubakari, A, and Duodu, A. (2014) An Evaluation of the Performance of DF in Cooperative MIMO Networks using Relay Strategies, in Proc. International Journal of Mobile Network Design and Innovation, 5 (3) 152-156.

23. Ashwin R. Jadhav, and Shankar, T. (2017) Whale optimization based energy-efficient cluster head selection algorithm for wireless sensor networks. arXiv preprint arXiv: 1711.09389 .

24. Sackey, Samson Hansen, James Adu Ansere, Joseph Henry Anajemba, Mohsin Kamal, and Celestine Iwendi. (2019) Energy Efficient Clustering Based Routing Technique in WSN using Brain Storm Optimization. In 2019 15th International Conference on Emerging Technologies, (ICET), 1-6. IEEE.

25. Maheshwari, Prachi, Ajay K. Sharma, and Karan Verma. (2020) Energy efficient cluster based routing protocol for WSN using butterfly optimization algorithm and ant colony optimization. Ad Hoc Networks, 110: 102317.

26. Wang, A, Yang, D, Sun, D. (2012) A clustering algorithm based on energy information and cluster heads expectation for wireless sensor networks. Computers \& Electrical Engineering, 38 (3) 662-671. 